\title{
ONE-BIT DOA ESTIMATION VIA SPARSE LINEAR ARRAYS
}

\author{
Saeid Sedighi ${ }^{\dagger}$, Bhavani Shankar ${ }^{\dagger}$, Mojtaba Soltanalian ${ }^{\ddagger}$, and Björn Ottersten ${ }^{\dagger}$ \\ ${ }^{\dagger}$ Interdisciplinary Centre for Security, Reliability and Trust (SnT), University of Luxembourg \\ $\ddagger$ Dept. of Electrical and Computer Engineering, University of Illinois at Chicago (UIC) \\ E-mails: \{saeid.sedighi,bhavani.shankar,bjorn.ottersten\}@uni.lu,msol@uic.edu
}

\begin{abstract}
Parameter estimation from noisy and one-bit quantized data has become an important topic in signal processing, as it offers low cost and low complexity in the implementation. On the other hand, Direction-of-Arrival (DoA) estimation using Sparse Linear Arrays (SLAs) has recently gained considerable interest in array processing due to their attractive capability of providing enhanced degrees of freedom. In this paper, the problem of DoA estimation from one-bit measurements received by an SLA is considered and a novel framework for solving this problem is proposed. The proposed approach first provides an estimate of the received signal covariance matrix through minimization of a constrained weighted least-squares criterion. Then, MUSIC is applied to the spatially smoothed version of the estimated covariance matrix to find the DoAs of interest. Several numerical results are provided to demonstrate the superiority of the proposed approach over its counterpart already propounded in the literature.
\end{abstract}

Index Terms - One-bit quantization, Sparse linear arrays, direction of arrival (DoA) estimation, weighted least-squares.

\section{INTRODUCTION}

The problem of Direction of Arrival (DoA) estimation is of central importance in the field of array processing with many applications in radar, sonar, and wireless communications [1-3]. Estimating DoAs using Uniform Linear Arrays (ULAs) is well investigated in the literature; a number of algorithms such as Maximum Likelihood (ML) estimation, MUSIC, ESPRIT and subspace fitting have been presented and their performance thoroughly analyzed [4-6]. However, it is widely known that ULAs are not capable of identifying more sources than the number of physical elements in the array $[2,6]$.

To transcend this limitation, exploitation of Sparse Linear Arrays (SLAs) with particular geometries, such as Minimum Redundancy Arrays (MRAs) [7], co-prime arrays [8] and nested arrays [9] has been proposed. These architectures can dramatically boost the degrees of freedom of the array for uncorrelated source signals such that a significantly larger number of sources than the number of physical elements in the array can be identified. In addition, the enhanced degrees of freedom provided by these SLAs can improve the resolution performance appreciably compared to ULAs [9]. These features have spurred further research on DoA estimation using SLAs in recent years. A detailed study on DoA estimation via

This work is partially supported by supported by the Luxembourg National Research Fund (FNR) under the ACCORDION project (reference number 11228830) and the BRIDGES project AWARDS as well as ERC AGNOSTIC ( Grant ID: 742648), NSF 1704401, 1809225, and a DPI seed award.
SLAs through an analysis of the Cramér-Rao Bound (CRB) has been conducted in [10]. Further, several approaches for DoA estimation via SLAs have been proposed in the literature. In general, existing estimators can be classified under two main groups: 1 . Sparsity-Based Methods (SBMs); 2. Augmented Covariance-Based Methods (ACBMs). SBMs first discretize the angular domain into a grid and then estimate DoAs by imposing sparsity constraints on source profiles and exploiting the compressive sensing recovery techniques [11-13]. In the second approach, DoAs are estimated by applying conventional subspace methods such as MUSIC, ESPRIT on an Augmented Sample Covariance Matrix (ASCM) obtained from the original sample covariance matrix by exploiting the difference coarray structure $[9,14,15]$.

Quantization of signals of interest is an essential step in digital signal processing. While high-resolution amplitude quantization at the Nyquist rate is typically desired, it may either be impractical or be impossible in many applications due to limitations on power consumption and production cost of Analog-to-Digital Converters (ADCs) [16]. One-bit quantizers allow for an extremely high sampling rate at a low cost and low power consumption while maintaining good performance. Recently, the use of one-bit quantized data has gained considerable interest in different applications such as massive MIMO systems [17-19], radar [20, 21] and array processing [22-26]. Using one-bit quantized data for DoA estimation has been considered in [22-25] for ULAs and it has been shown that one-bit quantization leads to a moderate performance loss compared to the case where unquantized data is used. Further, the problem of DoA estimation via SLAs and one-bit data has been addressed in [26]; it has been demonstrated that the performance degradation due to one-bit quantization can, to some extent, be compensated using SLAs.

In this paper, we propose a new framework for DoA estimation via SLAs using one-bit quantized measurements. Contrary to [26] which uses the Bussgang theorem, here the covaraince matrix of unquantized data is recovered from one-bit measurements through the solution of a constrained optimization problem. Then, by providing representative numerical results, it is shown that the estimated covariance matrix from the proposed approach leads to a better performance compared to the Bussgang-aided method given in [26].

Organization: Section 2 describes the system model. The problem formulation is given in Section 3. Section 4 provides the proposed algorithm for DoA estimation from one-bit measurements. The simulation results and related discussions are included in Section 5. Finally, Section 6 concludes the paper.

Notation: Vectors and matrices are referred to by lower- and upper-case bold-face, respectively. The superscripts $*, T, H$ denote the conjugate, transpose and Hermitian (conjugate transpose) operations, respectively. $\|\mathbf{A}\|_{F}$ stands for the Frobenius norm of $\mathbf{A}$. 
$[\mathbf{a}]_{i}$ indicates the $i^{\text {th }}$ entry of $\mathbf{a} . \hat{\mathbf{A}}$ and $\hat{\mathbf{a}}$ denote the estimate of $\mathbf{A}$ and $\mathbf{a}$, respectively. $\left(a_{1}, a_{2}, \cdots, a_{n}\right)$ is an $n$-tuple with elements of $a_{1}, a_{2}, \cdots, a_{n} .|\mathbb{A}|$ represents the cardinality of the set $\mathbb{A} \cdot \operatorname{diag}(\mathbf{a})$ is a diagonal matrix whose diagonal entries are equal to the elements of $\mathbf{a}$. The $M \times M$ identity matrix is denoted by $\mathbf{I}_{M}$. $(x)$ denotes the sign function with $(x)=1$ for $x \geq 0$ and $(x)=-1$ otherwise. The real and image part of $a$ are denoted by $\operatorname{Re}\{a\}$ and $\operatorname{Im}\{a\}$, respectively. $\mathbb{E}\{$.$\} stands for the statistical expectation. \otimes$ and $\odot$ represent Kronecker and Khatri-Rao products, respectively. $\operatorname{tr}(\mathbf{y})$, $\operatorname{rank}(\mathbf{A})$ and $\operatorname{vec}(\mathbf{A})=\left[\begin{array}{llll}\mathbf{a}_{1}^{T} & \mathbf{a}_{2}^{T} & \cdots & \mathbf{a}_{n}^{T}\end{array}\right]^{T}$ denote the trace, rank and vectorization operations, respectively. $\mathbf{A}^{\dagger}$ and indicates the pseudoinverse and of the full column rank matrix $\mathbf{A}$.

\section{SYSTEM MODEL}

We consider an SLA with $M$ elements located at positions $\left(m_{1} \frac{\lambda}{2}\right.$, $\left.m_{2} \frac{\lambda}{2}, \cdots, m_{M} \frac{\lambda}{2}\right)$ with $m_{i} \in \mathbb{M}$. Here $\mathbb{M}$ is a set of integers with cardinality $|\mathrm{M}|=M$, and $\lambda$ denotes the wavelength of the incoming signals. It is assumed $K$ narrowband signals with distinct DoAs $\boldsymbol{\theta}=\left[\theta_{1}, \theta_{2}, \cdots, \theta_{K}\right]^{T}$ impinge on the SLA from far field. While estimation of the number of sources is an important problem, we assume perfect knowledge of the number of sources here. The unquantized array measurements at time instance $t$ can be modeled as

$$
\mathbf{y}(t)=\mathbf{A}(\boldsymbol{\theta}) \mathbf{s}(t)+\mathbf{n}(t) \in \mathbb{C}^{M \times 1}, \quad t=1, \cdots, N,
$$

where $\mathbf{s}(t) \in \mathbb{C}^{K \times 1}$ denotes the vector of source signals, $\mathbf{n}(t) \in$ $\mathbb{C}^{M \times 1}$ is additive noise, and $\mathbf{A}(\boldsymbol{\theta})=\left[\mathbf{a}\left(\theta_{1}\right), \mathbf{a}\left(\theta_{2}\right), \cdots \mathbf{a}\left(\theta_{K}\right)\right] \in$ $\mathbb{C}^{M \times K}$ represents the SLA steering matrix with

$$
\mathbf{a}\left(\theta_{i}\right)=\left[\begin{array}{llll}
e^{\mathbf{j} \pi \sin \theta_{i} m_{1}} & e^{\mathrm{j} \pi \sin \theta_{i} m_{2}} & \cdots & e^{\mathrm{j} \pi \sin \theta_{i} m_{M}}
\end{array}\right]^{T},
$$

being the SLA manifold vector for the $i^{\text {th }}$ signal. Further, the following assumptions are made on source signals and noise:

A1 $\mathbf{n}(t)$ follows a zero-mean circular complex Gaussian distribution with the covariance matrix $\mathbb{E}\left\{\mathbf{n}(t) \mathbf{n}^{H}(t)\right\}=\sigma^{2} \mathbf{I}_{M}$.

A2 The source signal vector is modeled as a zero-mean circular complex Gaussian random vector with covariance matrix $\mathbb{E}\left\{\mathbf{s}(t) \mathbf{s}^{H}(t)\right\}=\operatorname{diag}(\mathbf{p})$ where $\mathbf{p}=\left[p_{1}, p_{2}, \cdots p_{K}\right]^{T} \in$ $\mathbb{R}_{>0}^{K \times 1}$ (i. e., $p_{l}>0 \forall l$ ).

A3 Source and noise vectors are mutually independent.

A4 There is no temporal correlation between the snapshots, i.e., $\mathbb{E}\left\{\mathbf{n}\left(t_{1}\right) \mathbf{n}^{H}\left(t_{2}\right)\right\}=\mathbb{E}\left\{\mathbf{s}\left(t_{1}\right) \mathbf{s}^{H}\left(t_{2}\right)\right\}=\mathbf{0}$ when $t_{1} \neq t_{2}$ and $\mathbf{0}$ is an all zero matrix of appropriate dimensions.

Based on the above assumptions, the covariance matrix of $\mathbf{y}(t)$ is expressed as

$$
\mathbf{R}=\mathbb{E}\left\{\mathbf{y}(t) \mathbf{y}^{H}(t)\right\}=\mathbf{A}(\boldsymbol{\theta}) \operatorname{diag}(\mathbf{p}) \mathbf{A}^{H}(\boldsymbol{\theta})+\sigma^{2} \mathbf{I}_{M} \in \mathbb{C}^{M \times M}
$$

Following $[9,10,14]$, the difference co-array model of the SLA is obtained by vectorizing the covariance matrix, which results in

$$
\begin{aligned}
\operatorname{vec}(\mathbf{R}) & =\left(\mathbf{A}^{*}(\boldsymbol{\theta}) \odot \mathbf{A}(\boldsymbol{\theta})\right) \mathbf{p}+\sigma^{2} \operatorname{vec}\left(\mathbf{I}_{M}\right), \\
& =\mathbf{J A}_{d}(\boldsymbol{\theta}) \mathbf{p}+\sigma^{2} \operatorname{vec}\left(\mathbf{I}_{M}\right) \in \mathbb{C}^{M^{2} \times 1},
\end{aligned}
$$

where $\mathbf{A}_{d}(\boldsymbol{\theta}) \in \mathbb{C}^{(2 D-1) \times K}$ is the steering matrix of the difference co-array whose elements are located at $\left(-\ell_{D-1} \frac{\lambda}{2}, \cdots, 0, \cdots, \ell_{D-1} \frac{\lambda}{2}\right)$ with $\ell_{i} \in \mathbb{D}=\left\{\left|m_{p}-m_{q}\right| \mid m_{p}, m_{q} \in \mathbb{M}\right\}$ and $D=|\mathbb{D}|$. Further, the selection matrix $\mathbf{J}$ is defined as follows

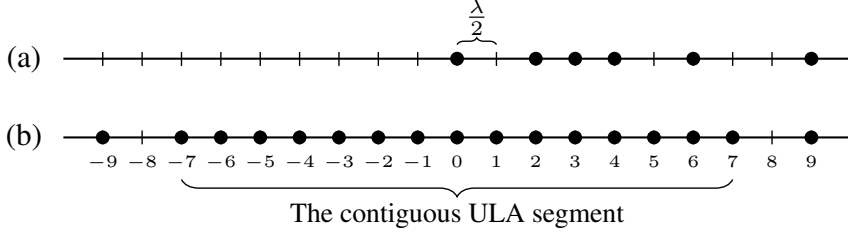

Fig. 1. Array geometry of a co-prime array with $M=6$ elements: (a) physical array with $\mathrm{M}=\{0,2,3,4,6,9\}$; (b) difference coarray with $\mathbb{D}=\{0,1,2,3,4,5,6,7,9\}$.

Definition 1. The binary matrix $\mathbf{J} \in\{0,1\}^{M^{2} \times(2 D-1)}$ is defined as [10]

$$
\mathbf{J}=\left[\begin{array}{lllll}
\operatorname{vec}\left(\mathbf{L}_{D-1}^{T}\right) & \cdots & \operatorname{vec}\left(\mathbf{L}_{0}\right) & \cdots & \operatorname{vec}\left(\mathbf{L}_{D-1}\right),
\end{array}\right],
$$

where $\left[\mathbf{L}_{n}\right]_{p, q}=\left\{\begin{array}{cc}1, & \text { if } m_{p}-m_{q}=\ell_{n}, \\ 0, & \text { otherwise, }\end{array}\right.$ with $1 \leq p, q \leq M$ and $0 \leq n \leq D-1$.

The difference co-array model in (4) can be perceived to be the response of a virtual array whose steering matrix is given by $\mathbf{A}_{d}(\boldsymbol{\theta})$ to the parameter vector with signal powers $\mathbf{p}$ in presence of the noise vector $\sigma^{2} \operatorname{vec}\left(\mathbf{I}_{M}\right)$. It has been shown in [8-10] that by properly designing SLAs and exploiting the resulting structure of the source signal covariance matrix efficiently, these arrays are capable of identifying more sources than the number of physical elements in the array. An illustrative example of an SLA, the corresponding difference co-array and its contiguous ULA segment is provided in Fig. 1.

In practice, the array measurements are quantized by ADCs. In the most extreme form of quantization, the measurements are directly converted into binary data by a comparator measuring the sign of the real and imaginary parts of the received signal. One-bit quantization allows for an extremely high sampling rate at a low cost and low power consumption. Assuming one-bit ADCs are used at the SLA, the quantized one-bit measurements are denoted as [17, 27]

$$
\mathbf{z}(t)=Q(\mathbf{y}(t)),
$$

where the $i^{\text {th }}$ element of $Q(\mathbf{y}(t))$ is given by $[Q(\mathbf{y}(t))]_{i}=$ $\frac{1}{\sqrt{2}}\left(\operatorname{Re}\left\{[\mathbf{y}(t)]_{i}\right\}\right)+\frac{\mathrm{j}}{\sqrt{2}}\left(\operatorname{Im}\left\{[\mathbf{y}(t)]_{i}\right\}\right)$. In this paper, we provide an algorithm to estimate DoAs from $\mathbf{z}(t)$.

\section{PROBLEM FORMULATION}

In this section, we formulate an optimization problem from which the covariance matrix of $\mathbf{y}(t)$, i.e., $\mathbf{R}$, can be recovered from one-bit observations. Once $\mathbf{R}$ is obtained, Co-Array-Based MUSIC (CABMUSIC), described in $[9,14]$, can be used to estimate DoAs. It can be readily checked that $\mathbf{R}$ is a structured matrix with only $2 D-1$ free parameters, i.e.,

$$
\mathbf{R}=u_{0} \mathbf{L}_{0}+\sum_{i=1}^{D-1} u_{i} \mathbf{L}_{i}+\sum_{i=1}^{D-1} u_{i}^{*} \mathbf{L}_{i}^{T}
$$

where $u_{0}=\sum_{k=1}^{K} p_{k}+\sigma^{2}$ and $u_{i}=\sum_{k=1}^{K} p_{k} e^{\mathrm{j} \pi \sin \theta_{k} \ell_{i}}$. Hence, to estimate $\mathbf{R}$, we need only to estimate the complex vector $\mathbf{u}=$ $\left[u_{0}, u_{1}, \cdots, u_{D-1}\right]^{T}$. In case the unquantized data are available, minimization of the following weighted least squares criterion yields 
a large-snapshot maximum likelihood (ML) estimate of the structured covariance matrix in (7) $[28,29]$ :

$$
\begin{aligned}
& \left(\operatorname{vec}\left(\mathbf{Y} \mathbf{Y}^{H}\right)-\operatorname{vec}(\mathbf{R})\right)^{H}\left(\mathbf{R}^{-T} \otimes \mathbf{R}^{-1}\right)\left(\operatorname{vec}\left(\mathbf{Y} \mathbf{Y}^{H}\right)-\operatorname{vec}(\mathbf{R})\right) \\
= & \left\|\mathbf{R}^{-\frac{1}{2}}\left(\mathbf{Y} \mathbf{Y}^{H}-\mathbf{R}\right) \mathbf{R}^{-\frac{1}{2}}\right\|_{F}^{2},
\end{aligned}
$$

where $\mathbf{Y}=\frac{1}{\sqrt{N}}\left[\begin{array}{lll}\mathbf{y}(1) & \cdots & \mathbf{y}(N)\end{array}\right]$. Unfortunately, the above criterion is non-convex in $\mathbf{R}$. Hence, inspired by (8), the following alternative convex criterion was proposed in [30]

$$
\begin{aligned}
& \left\|\mathbf{R}^{-\frac{1}{2}}\left(\mathbf{Y} \mathbf{Y}^{H}-\mathbf{R}\right)\left(\mathbf{Y} \mathbf{Y}^{H}\right)^{-\frac{1}{2}}\right\|_{F}^{2} \\
& =\operatorname{tr}\left(\mathbf{R}^{\frac{1}{2}}\left(\mathbf{Y} \mathbf{Y}^{H}\right)^{-1} \mathbf{R}^{\frac{1}{2}}\right)+\operatorname{tr}\left(\mathbf{R}^{-\frac{1}{2}}\left(\mathbf{Y} \mathbf{Y}^{H}\right) \mathbf{R}^{-\frac{1}{2}}\right),
\end{aligned}
$$

which is convex in $\mathbf{R}$. The above objective function is shown to converge to the ML criterion for a growing number of snapshots. Using the objective function (9), an optimization problem for the covariance matrix recovery from the one-bit measurements can be cast as follows:

$$
\begin{array}{ll}
\underset{\mathbf{u}, \mathbf{Y}}{\operatorname{minimize}} & \operatorname{tr}\left(\mathbf{R}^{\frac{1}{2}}\left(\mathbf{Y} \mathbf{Y}^{H}\right)^{-1} \mathbf{R}^{\frac{1}{2}}\right)+\operatorname{tr}\left(\mathbf{R}^{-\frac{1}{2}}\left(\mathbf{Y} \mathbf{Y}^{H}\right) \mathbf{R}^{-\frac{1}{2}}\right) \\
\text { subject to } & \mathbf{R} \succ \mathbf{0}, \\
& \operatorname{vec}(\operatorname{Re}\{\mathbf{Z}\}) \odot \operatorname{vec}(\operatorname{Re}\{\mathbf{Y}\}) \succeq \mathbf{0}, \\
& \operatorname{vec}(\operatorname{Im}\{\mathbf{Z}\}) \odot \operatorname{vec}(\operatorname{Im}\{\mathbf{Y}\}) \succeq \mathbf{0},
\end{array}
$$

where $\mathbf{Z}=\left[\begin{array}{lll}\mathbf{z}(1) & \cdots & \mathbf{z}(N)\end{array}\right]$. The above optimization problem is non-convex due to its dependence on $\mathbf{Y}$, but it can be recast as a Semi-Definite Programming (SDP) by introducing some slack variables.

\section{ONE-BIT DOA ESTIMATION}

We consider the slack variables $\mathbf{X}, \mathbf{W}, \mathbf{T}$ and $\boldsymbol{\Phi}$ such that $\mathbf{X}=$ $\mathbf{R}^{\frac{1}{2}}\left(\mathbf{Y} \mathbf{Y}^{H}\right)^{-1} \mathbf{R}^{\frac{1}{2}}, \mathbf{W} \succeq \mathbf{X}^{-1}, \mathbf{T}=\mathbf{Y} \mathbf{Y}^{H}$ and $\boldsymbol{\Phi}=\mathbf{R}^{\frac{1}{2}}$. Then it can be shown that optimization problem (10) is equivalent to the following one

$$
\begin{array}{cl}
\underset{\begin{array}{c}
\text { u,Y,Xinize }, \mathbf{X}, \mathbf{\Phi} \\
\text { subject to }
\end{array}}{\operatorname{mini}} & \operatorname{tr}(\mathbf{X})+\operatorname{tr}(\mathbf{W}) \\
& \mathbf{R} \succ \mathbf{0}, \\
& {\left[\begin{array}{cc}
\mathbf{X} & \mathbf{I}_{M} \\
\mathbf{I}_{M} & \mathbf{W}
\end{array}\right] \succeq \mathbf{0},} \\
& \operatorname{vec}(\operatorname{Re}\{\mathbf{Z}\}) \odot \operatorname{vec}(\operatorname{Re}\{\mathbf{Y}\}) \succeq \mathbf{0}, \\
& \operatorname{vec}(\operatorname{Im}\{\mathbf{Z}\}) \odot \operatorname{vec}(\operatorname{Im}\{\mathbf{Y}\}) \succeq \mathbf{0}, \\
& \mathbf{X}=\mathbf{\Phi} \mathbf{T}^{-1} \mathbf{\Phi}, \\
& \mathbf{R}=\mathbf{\Phi} \mathbf{\Phi}, \\
& \mathbf{T}=\mathbf{Y} \mathbf{Y}^{H} .
\end{array}
$$

Optimization problem (11) is an SDP with three equality constraints, which are non-convex. It is however possible to replace the equality constraints in (11) with three rank constraints on semi-definite matrices using the following Lemma.

Lemma 1. Let $\mathbf{C}_{1} \in \mathbb{R}^{m \times m}, \mathbf{C}_{2} \in \mathbb{R}^{n \times n}$ and $\mathbf{C}_{12} \in \mathbb{R}^{m \times n}$. If $\mathbf{C}_{1} \succ \mathbf{0}$, then the equality $\mathbf{C}_{2}=\mathbf{C}_{12}^{H} \mathbf{C}_{1}^{-1} \mathbf{C}_{12}$ is equivalent to the following rank and semi-definite inequalities

$$
\operatorname{rank}\left(\left[\begin{array}{cc}
\mathbf{C}_{1} & \mathbf{C}_{12} \\
\mathbf{C}_{12}^{H} & \mathbf{C}_{2}
\end{array}\right]\right) \leq m \text { and }\left[\begin{array}{cc}
\mathbf{C}_{1} & \mathbf{C}_{12} \\
\mathbf{C}_{12}^{H} & \mathbf{C}_{2}
\end{array}\right] \succeq 0
$$

Proof. It is readily confirmed that $\mathbf{C}_{2}=\mathbf{C}_{12}^{H} \mathbf{C}_{1}^{-1} \mathbf{C}_{12}$ if and only if $\operatorname{rank}\left(\mathbf{C}_{2}-\mathbf{C}_{12}^{H} \mathbf{C}_{1}^{-1} \mathbf{C}_{12}\right)=0$. Since $\mathbf{C}_{1}$ is positive definite, $\operatorname{rank}\left(\mathbf{C}_{2}-\mathbf{C}_{12}^{H} \mathbf{C}_{1}^{-1} \mathbf{C}_{12}\right)=0$ can be equivalently expressed as $\operatorname{rank}\left(\mathbf{C}_{1}\right)+\operatorname{rank}\left(\mathbf{C}_{2}-\mathbf{C}_{12}^{H} \mathbf{C}_{1}^{-1} \mathbf{C}_{12}\right) \leq m$. Further, it follows from the Guttman rank additivity formula [31] that $\operatorname{rank}\left(\mathbf{C}_{1}\right)+\operatorname{rank}\left(\mathbf{C}_{2}-\mathbf{C}_{12}^{H} \mathbf{C}_{1}^{-1} \mathbf{C}_{12}\right)=\operatorname{rank}\left(\left[\begin{array}{cc}\mathbf{C}_{1} & \mathbf{C}_{12} \\ \mathbf{C}_{12}^{H} & \mathbf{C}_{2}\end{array}\right]\right)$. This gives the rank condition in (12). Moreover, it follows from $\mathbf{C}_{2}-\mathbf{C}_{12}^{H} \mathbf{C}_{1}^{-1} \mathbf{C}_{12}=0$ and $\mathbf{C}_{1} \succ \mathbf{0}$ that $\left[\begin{array}{cc}\mathbf{C}_{1} & \mathbf{C}_{12} \\ \mathbf{C}_{12}^{H} & \mathbf{C}_{2}\end{array}\right]$ has to be positive semi-definite

By making use of Lemma 1, it is possible to recast optimization problem (11) as follows:

$$
\begin{array}{cl}
\underset{\mathbf{u}, \mathbf{Y}, \mathbf{X}, \mathbf{W}, \mathbf{T}, \mathbf{\Phi}}{\operatorname{minimize}} \text { subject to } & \operatorname{tr}(\mathbf{X})+\operatorname{tr}(\mathbf{W}) \\
& \mathbf{R} \succ \mathbf{0}, \\
& {\left[\begin{array}{cc}
\mathbf{X} & \mathbf{I}_{M} \\
\mathbf{I}_{M} & \mathbf{W}
\end{array}\right] \succeq \mathbf{0},} \\
& \operatorname{vec}(\operatorname{Re}\{\mathbf{Z}\}) \odot \operatorname{vec}(\operatorname{Re}\{\mathbf{Y}\}) \succeq \mathbf{0}, \\
& \operatorname{vec}(\operatorname{Im}\{\mathbf{Z}\}) \odot \operatorname{vec}(\operatorname{Im}\{\mathbf{Y}\}) \succeq \mathbf{0}, \\
& {\left[\begin{array}{cc}
\mathbf{T} & \mathbf{\Phi} \\
\mathbf{\Phi} & \mathbf{X}
\end{array}\right] \succeq \mathbf{0},} \\
& {\left[\begin{array}{cc}
\mathbf{I}_{M} & \mathbf{\Phi} \\
\mathbf{\Phi} & \mathbf{R}
\end{array}\right] \succeq \mathbf{0},} \\
& {\left[\begin{array}{cc}
\mathbf{I}_{N} & \mathbf{Y}^{H} \\
\mathbf{Y} & \mathbf{T}
\end{array}\right] \succeq \mathbf{0},} \\
& \operatorname{rank}\left(\left[\begin{array}{cc}
\mathbf{T} & \mathbf{\Phi} \\
\mathbf{\Phi} & \mathbf{X}
\end{array}\right]\right) \leq M, \\
& \left.\operatorname{rank}\left(\begin{array}{cc}
\mathbf{I}_{M} & \mathbf{\Phi} \\
\boldsymbol{\Phi}^{H} & \mathbf{R}
\end{array}\right]\right) \leq M, \\
& \operatorname{rank}\left(\left[\begin{array}{cc}
\mathbf{I}_{N} & \mathbf{Y} \\
\mathbf{Y} & \mathbf{T}
\end{array}\right]\right) \leq N .
\end{array}
$$

The above optimization problem is an SDP with three rank constraints on semi-definite matrices, which can be solved iteratively using Algorithm 1 where the sequential problem at iteration $k$ is formulated as

$$
\begin{aligned}
& \underset{\mathbf{u}_{k}, \mathbf{Y}_{k}, \mathbf{X}_{k}, \mathbf{W}_{k}, \mathbf{T}_{k}, \mathbf{\Phi}_{k}, e_{k}}{\operatorname{minimize}} \operatorname{tr}\left(\mathbf{X}_{k}\right)+\operatorname{tr}\left(\mathbf{W}_{k}\right)+w_{k} e_{k} \\
& \text { subject to } \quad \mathbf{R}_{k} \succ \mathbf{0} \text {, } \\
& {\left[\begin{array}{ll}
\mathbf{X}_{k} & \mathbf{I}_{M} \\
\mathbf{I}_{M} & \mathbf{W}_{k}
\end{array}\right] \succeq \mathbf{0},} \\
& \operatorname{vec}(\operatorname{Re}\{\mathbf{Z}\}) \odot \operatorname{vec}\left(\operatorname{Re}\left\{\mathbf{Y}_{k}\right\}\right) \succeq \mathbf{0} \text {, } \\
& \operatorname{vec}(\operatorname{Im}\{\mathbf{Z}\}) \odot \operatorname{vec}\left(\operatorname{Im}\left\{\mathbf{Y}_{k}\right\}\right) \succeq \mathbf{0},
\end{aligned}
$$

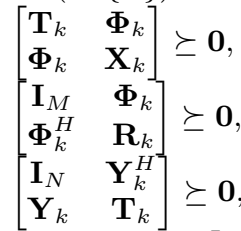

$$
\begin{aligned}
& e_{k} \mathbf{I}_{M}-\mathbf{V}_{k-1}^{H}\left[\begin{array}{ll}
\mathbf{T}_{k} & \boldsymbol{\Phi}_{k} \\
\boldsymbol{\Phi}_{k} & \mathbf{X}_{k}
\end{array}\right] \mathbf{V}_{k-1} \succeq \mathbf{0}, \\
& e_{k} \mathbf{I}_{M}-\mathbf{F}_{k-1}^{H}\left[\begin{array}{ll}
\mathbf{I}_{M} & \boldsymbol{\Phi}_{k} \\
\mathbf{\Phi}_{k}^{H} & \mathbf{R}_{k}
\end{array}\right] \mathbf{F}_{k-1} \succeq \mathbf{0} \\
& e_{k} \mathbf{I}_{M}-\mathbf{G}_{k-1}^{H}\left[\begin{array}{ll}
\mathbf{I}_{N} & \mathbf{Y}_{k}^{H} \\
\mathbf{Y}_{k} & \mathbf{T}_{k}
\end{array}\right] \mathbf{G}_{k-1} \succeq \mathbf{0}, \\
& e_{k} \leq e_{k-1} \text {, }
\end{aligned}
$$

where $\mathbf{V}_{k-1}, \mathbf{F}_{k-1}$ and $\mathbf{G}_{k-1}$ are the eigenvectors corresponding to the $M$ smallest eigenvalues of $\left[\begin{array}{ll}\mathbf{T}_{k-1} & \mathbf{\Phi}_{k-1} \\ \boldsymbol{\Phi}_{k-1} & \mathbf{X}_{k-1}\end{array}\right],\left[\begin{array}{cc}\mathbf{I}_{M} & \boldsymbol{\Phi}_{k-1} \\ \boldsymbol{\Phi}_{k-1}^{H} & \mathbf{R}_{k-1}\end{array}\right]$ 
and $\left[\begin{array}{cc}\mathbf{I}_{N} & \mathbf{Y}_{k-1}^{H} \\ \mathbf{Y}_{k-1} & \mathbf{T}_{k-1}\end{array}\right]$ obtained at the previous iteration. Indeed, the optimization problem which should be solved at each step of Algorithm 1 is an SPD that can be solved efficiently. Further, to obtain $\mathbf{V}_{0}, \mathbf{F}_{0}$ and $\mathbf{G}_{0}$, it is possible to use the relaxed solution of (13) by dropping the rank constraints. It is proved in [32] that Algorithm 1 converges to at least a local minimum of (13). Once $\mathbf{R}$ is obtained from Algorithm 1, Co-Array-Based MUSIC (CAB-MUSIC), described in $[9,14]$, can be employed to estimate DoAs.

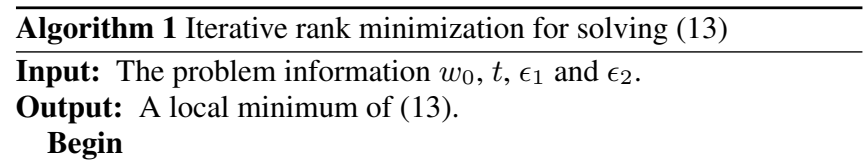

1. initialization: Set $k=0$. Solve the relaxed problem in (13) by dropping the rank constraints to obtain $\mathbf{V}_{0}, \mathbf{F}_{0}$ and $\mathbf{G}_{0}$.

2. while: $e_{k} \geq \epsilon_{1}$ and $\mid \operatorname{tr}\left(\mathbf{X}_{k}\right)+\operatorname{tr}\left(\mathbf{W}_{k}\right)-\operatorname{tr}\left(\mathbf{X}_{k-1}\right)+$ $\operatorname{tr}\left(\mathbf{W}_{k-1}\right) \mid \geq \epsilon_{2}$.

3. Solve the sequential problem (14).

4. Update $\mathbf{V}_{k-1}, \mathbf{F}_{k-1}$ and $\mathbf{G}_{k-1}$ and set $k=k+1$.

5. Update $w_{k}$ as $w_{k}=w_{k-1} * t$.

6. end while

End

\section{SIMULATION RESULTS}

In this section, we provide some numerical results to compare the performance of the proposed approach to that of the Bussgang-aided method given in [26]. In all experiments, each simulated point has been computed by 1000 Monte Carlo repetitions. In addition, it is assumed that the $K$ independent sources are located at $\left\{-60^{\circ}+\right.$ $\left.120^{\circ}(k-1) /(K-1) \mid k=0,1, \cdots, K-1\right\}$. All sources have an equal power, i.e., $p_{k}=p$ for all $k$, and the SNR is defined as $10 \log \frac{p}{\sigma^{2}}$. Throughout this section, we use a nested array with $M=$ 12 physical elements and the following geometry:

$$
\mathrm{M}_{\text {nested }}:\{1,2,3,4,5,6,7,14,21,28,35,42\} .
$$

Fig. 2 depicts the Root-Mean-Squares-Error (RMSE) for $\theta_{2}$ in degree versus SNR. The number of snapshots is considered to be $N=500$. Given $M=12$, two different scenarios are considered: (a) $K=3<M$, and (b) $K=14>M$. It is observed that the proposed approach presents a better performance compared to the Bussgang-aided method in both cases and shows an RMSE very close to that of DoA estimates obtained from the unquantized data. As an illustrative example, the proposed algorithm is able to improve the quantization loss, defined as $10 \log \left(\mathrm{MSE}_{\text {quantized }} / \mathrm{MSE}_{\text {unquantized }}\right)$, about 2 to $2.5 \mathrm{~dB}$ compared to the Bussgang-aided method at $\mathrm{SNR}=5 \mathrm{~dB}$. Fig. 3 plots the RMSE for $\theta_{2}$ in degree versus the number of snapshots for $\mathrm{SNR}=3 \mathrm{~dB}$ and $K=5$. Fig. 3 indicates that the proposed algorithm preforms very closely to the unquantized case when an adequate large number of snapshots is available. However, the performance of DoA estimation via one-bit measurements start deviating from the performance acquired using the unquantized data when the number of snapshots reduces.

\section{CONCLUSION}

A novel framework for estimating DoAs from one-bit measurements obtained by an SLA was proposed in this paper. The proposed algo-

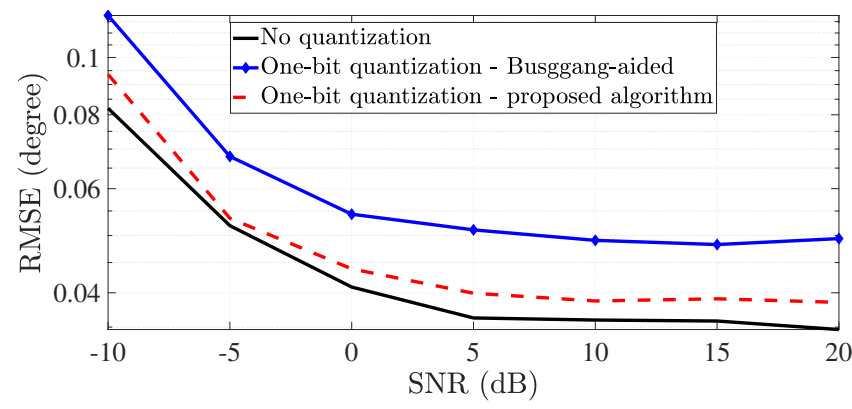

(a)

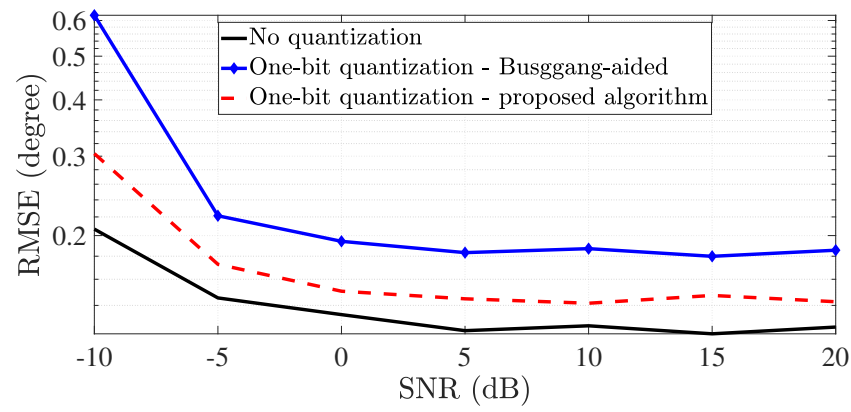

(b)

Fig. 2. RMSE in degree for $\theta_{2}$ versus SNR for a nested array with $M=12$ elements and configuration given in (15), $N=500$, and: (a) $K=3<M$; (b) $K=14>M$.

rithm provides an alternative approach to estimating the covaraince matrix of unquantized data beside Bussgang-aided approach already propounded in the literate. The provided simulation results showed that the proposed algorithm leads to better performance compared to the Bussgang-aided approach.

In the future, it is of considerable interest to investigate the onebit DoA estimation problem given in this paper when the thresholds at ADCs vary in time as well as across the array elements. Using the varying thresholds is expected to significantly enhance the performance of the proposed algorithm. In addition, it will be interesting to investigate the performance bounds of DoA estimation via SLAs and one-bit measurements. The computational complexity of the proposed techniques also need to be further evaluated.

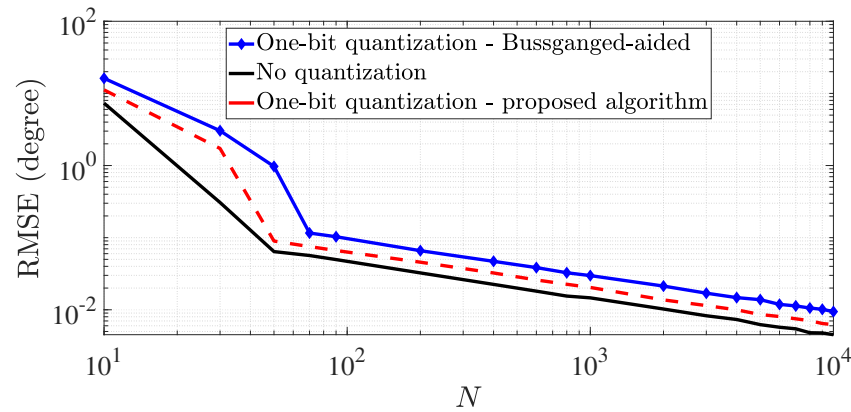

Fig. 3. RMSE in degree for $\theta_{2}$ versus the number of snapshots for a nested array with $M=12$ elements and configuration given in (15), $\mathrm{SNR}=0 \mathrm{~dB}$, and $K=5$. 


\section{REFERENCES}

[1] H. Van Trees, Optimum Array Processing (Detection, Estimation, and Modulation Theory, Part IV). New York: John Wiley and Sons Inc., 2002.

[2] S. S. Haykin, J. Litva, , and T. J. Shepherd, Eds., Radar Array Processing. Berlin, Germany: Springer-Verlag, 1993.

[3] B. Ottersten, "Array processing for wireless communications," in Proceedings of 8th Workshop on Statistical Signal and Array Processing, Jun 1996, pp. 466-473.

[4] A. Paulraj, B. Ottersten, R. Roy, A. Swindlehurst, G. Xu, and T. Kailath, "16 subspace methods for directions-of-arrival estimation," Handbook of Statistics, vol. 10, pp. 693-739, 1993.

[5] F. Li, H. Liu, and R. J. Vaccaro, "Performance analysis for daa estimation algorithms: unification, simplification, and observations," IEEE Trans. Aerosp. Electron. Syst, vol. 29, no. 4, pp. 1170-1184, Oct 1993.

[6] P. Stoica and A. Nehorai, "Performance study of conditional and unconditional direction-of-arrival estimation," IEEE Transactions on Acoustics, Speech, and Signal Processing, vol. 38, no. 10, pp. 1783-1795, Oct 1990.

[7] A. Moffet, "Minimum-redundancy linear arrays," IEEE Transactions on Antennas and Propagation, vol. 16, no. 2, pp. 172175, Mar 1968.

[8] P. P. Vaidyanathan and P. Pal, "Sparse sensing with co-prime samplers and arrays," IEEE Trans. Signal Process., vol. 59, no. 2, pp. 573-586, Feb 2011.

[9] P. Pal and P. P. Vaidyanathan, "Nested arrays: A novel approach to array processing with enhanced degrees of freedom," IEEE Transactions on Signal Processing, vol. 58, no. 8, pp. 4167-4181, Aug 2010.

[10] C. L. Liu and P. Vaidyanathan, "Cramér-Rao bounds for coprime and other sparse arrays, which find more sources than sensors," Digital Signal Processing, vol. 61, pp. 43 - 61, 2017.

[11] Y. D. Zhang, M. G. Amin, and B. Himed, "Sparsity-based DoA estimation using co-prime arrays," in 2013 IEEE International Conference on Acoustics, Speech and Signal Processing, May 2013, pp. 3967-3971.

[12] Z. Tan and A. Nehorai, "Sparse direction of arrival estimation using co-prime arrays with off-grid targets," IEEE Signal Processing Letters, vol. 21, no. 1, pp. 26-29, Jan 2014.

[13] Z. Yang, L. Xie, and C. Zhang, "A discretization-free sparse and parametric approach for linear array signal processing," IEEE Transactions on Signal Processing, vol. 62, no. 19, pp. 4959-4973, Oct 2014.

[14] M. Wang and A. Nehorai, "Coarrays, MUSIC, and the CramérRao bound," IEEE Trans. Signal Process., vol. 65, no. 4, pp. 933-946, Feb 2017.

[15] S. Sedighi, R. B. S. Mysore, and B. Ottersten, "A statistically efficient estimator for co-array based DoA estimation," in $52^{\text {nd }}$ Annual Asilomar Conference on Signals, Systems, and Computers, October 2018. [Online]. Available: http://hdl.handle.net/10993/37660

[16] R. H. Walden, "Analog-to-digital converter survey and analysis," IEEE Journal on Selected Areas in Communications, vol. 17, no. 4, pp. 539-550, April 1999.
[17] L. Lu, G. Y. Li, A. L. Swindlehurst, A. Ashikhmin, and R. Zhang, "An overview of massive MIMO: Benefits and challenges," IEEE Journal of Selected Topics in Signal Processing, vol. 8, no. 5, pp. 742-758, Oct 2014.

[18] S. Jacobsson, G. Durisi, M. Coldrey, U. Gustavsson, and C. Studer, "One-bit massive MIMO: Channel estimation and high-order modulations," in 2015 IEEE International Conference on Communication Workshop (ICCW), June 2015, pp. 1304-1309.

[19] S. Rao, A. Mezghani, and A. L. Swindlehurst, "Channel estimation in one-bit massive MIMO systems: Angular versus unstructured models," IEEE Journal of Selected Topics in Signal Processing, vol. 13, no. 5, pp. 1017-1031, Sep. 2019.

[20] A. Ameri, A. Bose, J. Li, and M. Soltanalian, "One-bit radar processing with time-varying sampling thresholds," IEEE Transactions on Signal Processing, vol. 67, no. 20, pp. 52975308, Oct 2019.

[21] J. Li, M. M. Naghsh, S. J. Zahabi, and M. Modarres-Hashemi, "Compressive radar sensing via one-bit sampling with timevarying thresholds," in 2016 50th Asilomar Conference on Signals, Systems and Computers, Nov 2016, pp. 1164-1168.

[22] O. Bar-Shalom and A. J. Weiss, "DoA estimation using onebit quantized measurements," IEEE Transactions on Aerospace and Electronic Systems, vol. 38, no. 3, pp. 868-884, July 2002.

[23] C. Stöckle, J. Munir, A. Mezghani, and J. A. Nossek, "1-bit direction of arrival estimation based on compressed sensing," in 2015 IEEE 16th International Workshop on Signal Processing Advances in Wireless Communications (SPAWC), June 2015, pp. 246-250.

[24] M. Stein, K. Barbe, and J. A. Nossek, "DoA parameter estimation with 1-bit quantization bounds, methods and the exponential replacement," in WSA 2016; 20th International ITG Workshop on Smart Antennas, March 2016, pp. 1-6.

[25] X. Huang and B. Liao, "One-bit MUSIC," IEEE Signal Processing Letters, vol. 26, no. 7, pp. 961-965, July 2019.

[26] C. Liu and P. P. Vaidyanathan, "One-bit sparse array DoA estimation," in 2017 IEEE International Conference on Acoustics, Speech and Signal Processing (ICASSP), March 2017, pp. 3126-3130.

[27] A. Ameri, J. Li, and M. Soltanalian, "One-bit radar processing and estimation with time-varying sampling thresholds," in 2018 IEEE 10th Sensor Array and Multichannel Signal Processing Workshop (SAM), July 2018, pp. 208-212.

[28] B. Ottersten, P. Stoica, and R. Roy, "Covariance matching estimation techniques for array signal processing applications," Digital Signal processing, vol. 8, no. 3, pp. 185-210, July 1998.

[29] T. Anderson, An introduction to multivariate statistical analysis, 3rd ed. New York: John Wiley and Sons Inc., 2003.

[30] P. Stoica, P. Babu, and J. Li, "New method of sparse parameter estimation in separable models and its use for spectral analysis of irregularly sampled data," IEEE Transactions on Signal Processing, vol. 59, no. 1, pp. 35-47, Jan 2011.

[31] M. S. Gowda and R. Sznajder, "Schur complements, Schur determinantal and Haynsworth inertia formulas in Euclidean Jordan algebras," Linear Algebra Appl, vol. 432, pp. 1553-1559, 2010.

[32] C. Sun and R. Dai, "Rank-constrained optimization and its applications," Automatica, vol. 82, pp. 128-136, 2017. 